\title{
Parton Hadron Quantum Molecular Dynamics (PHQMD) - a Novel Microscopic N-Body Transport Approach for Heavy-Ion Dynamics and Hypernuclei Production
}

\author{
E. Bratkovskaya ${ }^{1,2}$, J. Aichelin ${ }^{3,4}$, A. Le Fèvre ${ }^{1}$, V. Kireyeu ${ }^{5}$, V. Kolesnikov ${ }^{5}$, \\ Y. Leifels ${ }^{1}$, and and V. Voronyuk ${ }^{5}$ \\ ${ }^{1}$ GSI Helmholtzzentrum für Schwerionenforschung GmbH, Planckstr. 1, 64291 \\ Darmstadt, Germany \\ E.Bratkovskaya@gsi.de, \\ 2 Institut für Theoretische Physik, Johann Wolfgang Goethe-Universität, \\ Max-von-Laue-Str. 1, 60438 Frankfurt am Main, Germany \\ 3 SUBATECH, Université de Nantes, IMT Atlantique, IN2P3/CNRS 4 rue Alfred \\ Kastler, 44307 Nantes cedex 3, France \\ 4 Frankfurt Institute for Advanced Studies, Ruth Moufang Str. 1, 60438 Frankfurt, \\ Germany \\ 5 Joint Institute for Nuclear Research, Joliot-Curie 6, 141980 Dubna, Moscow region, \\ Russia
}

\begin{abstract}
We present the novel microscopic n-body dynamical transport approach PHQMD (Parton-Hadron-Quantum-Molecular-Dynamics) for the description of particle production and cluster formation in heavyion reactions at relativistic energies. The PHQMD extends the established PHSD (Parton-Hadron-String-Dynamics) transport approach by replacing the mean field by density dependent two body interactions in a similar way as in the Quantum Molecular Dynamics (QMD) models. This allows for the calculation of the time evolution of the n-body Wigner density and therefore for a dynamical description of clusters and hypernuclei formation. The clusters are identified with the MST (Minimum Spanning Tree) or the SACA (Simulated Annealing Cluster Algorithm) algorithm which - by regrouping the nucleons in single nucleons and noninteracting clusters - generates the most bound configuration of nucleons and clusters. Collisions among particles in PHQMD are treated in the same way as in PHSD. In Ref. 1] we presented the first results from the PHQMD for general 'bulk' observables such as rapidity distributions and transverse mass spectra for hadrons as well as for clusters production, including hypernuclei, at SIS and FAIR/NICA/BES RHIC energies. The selected results on clusters and hypernuclei production from Ref. 1] are discussed in this contribution.
\end{abstract}

Keywords: heavy-ion collisions, clusters production 


\section{Introduction}

The study of cluster and hypernucleus production, which reflects the phase space density during the expansion phase, is of particular interest from experimental as well as from theoretical side. The production mechanisms of hypernuclei may shed light on the theoretical understanding of the dynamical evolution of heavyion reactions which cannot be addressed by other probes. In particular, the formation of heavy projectile/target like hypernuclei elucidates the physics at the transition region between spectator and participant matter. Since hyperons are produced in the overlap region, multiplicity as well as rapidity distributions of hypernuclei formed in the target/projectile region depend crucially on the interactions of the hyperons with the hadronic matter, e.g. cross sections and potentials. On the other hand, midrapidity hypernuclei test the phase space distribution of baryons in the expanding participant matter, especially whether the phase space distributions of strange and non-strange baryons are similar and whether they are in thermal equilibrium.

The description of cluster and hypernuclei formation is a challenging theoretical task which requires I) the microscopic dynamical description of the time evolution of heavy-ion collisions; II) the modelling of the mechanisms for the clusters formation.

Cluster formation has often been described either by a coalescence model 203. or statistical methods 4 assuming that during the heavy-ion reaction at least a subsystem achieves thermal equilibration. Both of these models have serious drawbacks. The most essential is that they are not able to address the question of how the clusters are formed and what we can learn from the cluster formation about the reaction dynamics.

\section{PHQMD: basic ideas}

In order to overcome the limitations of existing models for the clusters formation, we advance the novel Parton-Hadron-Quantum-Molecular Dynamics (PHQMD) [1] approach which is based on the collision integrals of the Parton-Hadron-String Dynamics approach [5]6] and density dependent 2-body potential interactions of QMD type models $7 / 8$. These clusters can be identified by two methods: either by the minimum spanning tree (MST) procedure [7] or by a cluster finding algorithm based on the simulated annealing technique, the Simulated Annealing Clusterization Algorithm (SACA) 9 10. Presently an extended version - the Fragment Recognition In General Application (FRIGA) 11 is under development which includes symmetry and pairing energy as well as hyperon-nucleon interactions. The MST algorithm is based on spatial correlations and it is effective in finding the clusters at the end of the reaction. In order to identify the cluster formation already at early times of the reaction, when the collisions between the nucleons are still on-going and the nuclear density is high, the SACA approach is used. It is based on the idea of Dorso and Randrup [12] that the most bound configuration of nuclei and nucleons evolves in time towards the final cluster distribution. 


\section{Results}

First results from combined PHSD/SACA approach have been reported in [13]. Recently we presented the first results from the PHQMD approach on 'bulk' dynamics, covering the energy range from SIS to RHIC, as well as the results on dynamical cluster formation, including hypernuclei, based on the MST and SACA models [1]. In this contribution we show the selected results from Ref. [1.
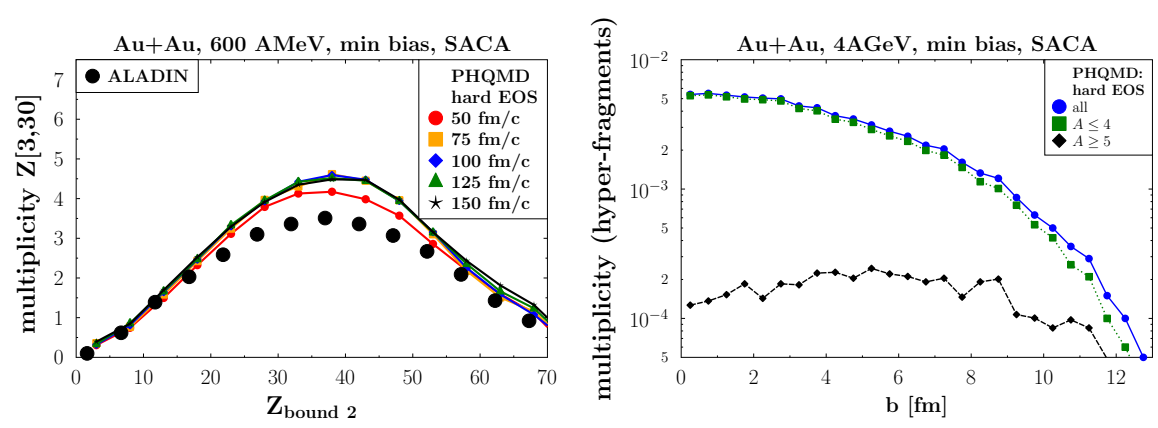

Fig. 1. (left) 'Rise and fall' of the multiplicity of clusters with $Z \in[3,30]$ as a function of the total bound charge $Z_{\text {bound }}$. Both quantities are measured for forward emitted clusters. The experimental data of the ALADIN Collaborations are from Ref. 14[11. The plot shows the PHQMD results with hard EoS using cluster identification by SACA for $600 \mathrm{AGeV}$ at different times - 50 (red line), 75(orange line), 100 (blue line), 125 (green line), 150 (black line) fm/c.

Fig. 2. (right) The multiplicity of hyperclusters as a function of the impact parameter for $\mathrm{Au}+\mathrm{Au}$ collisions at $4 \mathrm{AGeV}$ calculated with the PHQMD using the SACA cluster recognition algorithm. The blue dots show the multiplicity of all hyper-nuclei, while the green squares and black rhombus stand for $A \leq 4$ and $A \geq 5$, respectively.

In Fig. 1 we display our results for $\mathrm{Au}+\mathrm{Au}$ at $600 \mathrm{AMeV}$ calculated with a hard EoS in comparison with minimum bias ALADIN data [14. The clusters identified by SACA are stable for times larger than $50 \mathrm{fm} / \mathrm{c}$ as shown in Fig. 1. One can see clearly that PHQMD with a hard EoS reproduces quite nicely the experimentally observed 'rise and fall'. The rise and fall of the intermediate mass cluster multiplicity depends strongly on the nuclear equation-of-state. As shown in 1], the rise and fall for a soft EoS overpredicts the data at large $Z_{\text {bound 2. }}$. There in semi-peripheral and peripheral collisions, where $Z_{\text {bound } 2}$ is large, the spectator matter is much less stable and fragments into a much larger number of intermediate mass clusters as compared to a hard EoS (Fig. 1). Thus, the fragment pattern in semi-peripheral reactions can serve as an additional observable to determine the hadronic EoS experimentally.

A special interest is related to the production of hypernuclei in heavy-ion collisions. In Fig. 2 we show the multiplicity of light and heavy hypercluster as a 
function of the impact parameter for $\mathrm{Au}+\mathrm{Au}$ collisions at $4 \mathrm{AGeV}$. As seen from this figure, the yield of light hyper-clusters decreases with the impact parameter, mainly because the overlap region between projectile and target gets smaller and hence less hyperons are produced. In central collisions, mainly small hypernuclei $(A \leq 4)$ are formed while mid-central collisions are better suited for a study of heavier hypernuclei $(A \geq 5)$. Hypernuclei with $A \geq 5$ are dominantly produced by hyperons which enter the spectator matter and get caught there. Therefore, for heavy hyper-nuclei production there is a competition between the hyperon production which decreases with impact parameter and the spectator matter whose size increases with impact parameter.

Summarizing, we present the PHQMD transport approach which can be used for the dynamical cluster identification including the hypernuclei production from low to ultrarelativistic energies.

\section{Acknowledgements}

The authors acknowledge inspiring discussions with C. Blume, W. Cassing, C. Hartnack, P. Moreau, T. Song, Io. Vassiliev and M. Winn. Furthermore, we acknowledge support by the Deutsche Forschungsgemeinschaft (DFG, German Research Foundation), by the Russian Science Foundation grant 19-42-04101 and by the GSI-IN2P3 agreement under contract number 13-70. Also we thank the COST Action THOR, CA15213. The computational resources have been provided by the LOEWE-Center for Scientific Computing and the "Green Cube" at GSI.

\section{References}

1. J. Aichelin, E. Bratkovskaya, A. Le Fvre, V. Kireyeu, V. Kolesnikov, Y. Leifels and V. Voronyuk, arXiv:1907.03860 [nucl-th].

2. L. Zhu, C. M. Ko and X. Yin, Phys. Rev. C 92, 064911 (2015).

3. Z. Feckov, J. Steinheimer, B. Tomik and M. Bleicher, Phys. Rev. C 93, 054906 (2016).

4. A. S. Botvina, K. K. Gudima, J. Steinheimer, M. Bleicher and I. N. Mishustin, Phys. Rev. C 84, 064904 (2011).

5. W. Cassing and E. L. Bratkovskaya, Nucl. Phys. A 831, 215 (2009).

6. O. Linnyk, E. L. Bratkovskaya, W. Cassing, Prog. Part. Nucl. Phys. 87, 50 (2016).

7. J. Aichelin, Phys. Rept. 202, 233 (1991).

8. R. Marty and J. Aichelin, Phys. Rev. C 87, 034912 (2013).

9. R. K. Puri, C. Hartnack and J. Aichelin, Phys. Rev. C 54, R28 (1996).

10. R. K. Puri and J. Aichelin, J. Comput. Phys. 162, 245 (2000).

11. A. Le Fvre, J. Aichelin, C. Hartnack and Y. Leifels, Phys. Rev. C 100, 034904 (2019).

12. C. Dorso and J. Randrup, Phys. Lett. B 301, 328 (1993).

13. A. Le Fèvre, Y. Leifels, J. Aichelin, C. Hartnack, V. Kireyev and E. Bratkovskaya, J. Phys. Conf. Ser. 668, 012021 (2016).

14. A. Le Fèvre, Y. Leifels, J. Aichelin, C. Hartnack, V. Kireyev and E. Bratkovskaya, Nuovo Cim. C 39, 399 (2017). 